\title{
La relación entre enajenación y salud teniendo en cuenta la propuesta de los tres niveles de determinación social de la salud
}

The relationship between alienation and health taking into account the proposal of the three levels of social determination of health

Adriana Mederos González. ${ }^{1}$, Osleiys Orta Espinosa. ${ }^{2}$ \& Daniel Enrique Martin Morell ${ }^{3}$

\begin{abstract}
.
Alienation arises, when the product of man's work, instead of satisfying his needs, becomes something alien, that is, the product takes on a totally independent existence from the man who produced it, once that product gains its independence, Alienated work is generated, through which man becomes a slave more and more to the things he produces.

Alienation is a phenomenon that acquires its most deployed form of manifestation in

\section{Resumen.}

La alienación surge, cuando el producto del trabajo del hombre, en lugar de satisfacer sus necesidades, se vuelve algo ajeno, es decir el producto cobra una existencia totalmente independiente del hombre que fue quien la produjo, una vez que ese producto cobra su independencia, se genera el trabajo alienado, a través del cual el hombre se vuelve esclavo cada vez más y más de las cosas que produce. La enajenación es un fenómeno que adquiere su forma más desplegada de manifestación en

\footnotetext{
${ }^{1}$ Ciencias Médicas Mayabeque, Mayabeque, Cuba, amederos@infomed.sld.cu 0002-7353-0140

${ }^{2}$ Ciencias Médicas Mayabeque, Mayabeque, Cuba, osleorta@infomed.sld.cu 7353-0141 ${ }^{3}$ Ciencias Médicas Mayabeque, Mayabeque, Cuba, dmartin@infomed.sld.cu iD https://orcid.org/0000-00027353-0142

https://orcid.org/0000https://orcid.org/0000-0002-
} 
capitalism, where relations between men are established in the market and under this social capitalist division human relations appear as properties of commodities.

Keywords: Alignment, alienation, phenomenon. el capitalismo, donde las relaciones entre los hombres se establecen en el mercado y bajo esta división social capitalista las relaciones humanas aparecen como propiedades de las mercancías.

Palabras claves: Alineación, enajenación, fenómeno.

\section{Introducción.}

El hombre en su interacción con la naturaleza y la sociedad constantemente está creando valores materiales y espirituales para satisfacer sus necesidades. Proceso de transformación durante el cual el individuo, las distintas clases y grupos sociales reciben simultáneamente las influencias que ejerce la sociedad sobre ellos, nutriéndolos de experiencias, convicciones, conocimientos, actitudes, rasgos morales, normas de conducta, habilidades y maneras de concebir el mundo, lo cual permite un permanente perfeccionamiento de las relaciones sociales y de producción.

En la actualidad las relaciones sociales han llegado a tal punto, en que los individuos deben apropiarse de la totalidad existente de las fuerzas productivas, no sólo para lograr la auto actividad, sino también, para salvaguardar su propia existencia. (1) Proceso que se desarrolla cuando aparecen tensadas en grado máximo las contradicciones sociales y se expresan de disímiles formas las carencias y angustias de las mayorías.

Por esta razón se hace necesario abordar en primera instancia el tema referente al Humanismo (2), el cual se define como el proyecto emancipador de toda la sociedad, el dominio de sus miembros de la existencia propia en condiciones de igualdad y justicia, la dignificación del individuo y la práctica de un conjunto de modificaciones sucesivas que engloban la totalidad de la vida ciudadana. Pero a su vez también abordar el tema de la Enajenación o alienación como fenómeno contrapuesto del humanismo.

Pues si el humanismo promueve una vida plena donde el individuo es capaz de mantener la relación sujeto - objeto y transformar la realidad según sus necesidades a través de la práctica en la actividad humana, la Enajenación se define como el proceso mediante el cual se despoja al hombre de su carácter humano y se ve dominado por el mundo de objetos creados por él, y así en determinadas circunstancias históricas devienen hostiles a su desarrollo, o sea, es la pérdida de la capacidad del sujeto humano de dominar los productos de su actividad, de tal manera que estos productos se convierten en algo extraño a él que llega a dominarlo, haciendo que pierda su esencia humana. Es la dominación del objeto sobre el sujeto.

La alienación surge, cuando el producto del trabajo del hombre, en lugar de satisfacer sus necesidades, se vuelve algo ajeno, es decir el producto cobra una existencia totalmente independiente del hombre que fue quien la produjo, una vez que ese producto cobra su 
independencia, se genera el trabajo alienado, a través del cual el hombre se vuelve esclavo cada vez más y más de las cosas que produce.

La enajenación es un fenómeno que adquiere su forma más desplegada de manifestación en el capitalismo, donde las relaciones entre los hombres se establecen en el mercado y bajo esta división social capitalista las relaciones humanas aparecen como propiedades de las mercancías.

Cuanto más produce el trabajador, tanto menos ha de consumir; cuanto más valor crea, tanto más sin valor, tanto más indigno es él; cuanto más elaborado su producto, tanto más deforme el trabajador; cuanto más civilizado su objeto, tanto más bárbaro el trabajador; cuanto más rico espiritualmente se hace el trabajo, tanto más desespiritualizado y ligado a la naturaleza queda el trabajador.

Esto no quiere decir que en el socialismo no exista enajenación. Pues éste es un sistema social superior que se erige del capitalismo, por lo que hereda una estructura socioeconómica deformada, subdesarrollada y dependiente como la nuestra. Donde, aunque se trate de erradicar o eliminar todas las taras del pasado aún quedan vestigios del sistema capitalista en la sociedad. La enajenación en el socialismo se expresa en el orden político económico y social: en el orden político se refleja a través del ejercicio del poder estatal donde un ejemplo es la evidencia del burocratismo, defecto este heredado del capitalismo; en lo económico, la existencia de factores coyunturales adversos que traen consigo afectaciones y limitaciones de recursos los cuales frenan el desarrollo social y particularmente el consumo individual; y en el ámbito social a través de la insuficiente madurez del ser social y de la conciencia social e individual. Esto ocurre a pesar de que desde el triunfo revolucionario se dan pasos vertiginosos respecto a la educación del individuo como ser social capaz de desarrollar una conciencia social en este modo de producción, manteniendo así su esencia humana.

Por estas razones es de vital importancia el estudio de este tema ya que tiene que ver con la comprensión que se tenga sobre el hombre y su actividad, o sea, sobre su esencia humana y la cualidad de su relación con el mundo circundante.

Se plantea como objetivo:

Analizar desde un enfoque filosófico la relación entre enajenación y salud teniendo en cuenta la propuesta de los tres niveles de determinación social de la salud.

\section{Desarrollo}

Singular importancia tiene la concepción marxista sobre las causas de la enajenación de la esencia humana. Estas causas se hallan según Marx, en las condiciones concretas de vida, en la calidad de las relaciones sociales en la que el hombre se desenvuelve. Por tanto, la alienación tiene por causas unas determinadas condiciones materiales que constituyen factores enajenantes de la actividad vital humana y no sólo es "una ilusión del espíritu" como lo comprende el idealismo. De ahí el partidismo filosófico de la enajenación 
La explicación hegeliana como ejemplo de esta corriente filosófica limita la enajenación sólo al aspecto subjetivo; el hombre no "reconoce" en lo creado su propia obra, y por ende considera su propio producto como una realidad extraña. Por mucho que se profundice en la posición idealista sobre las causas de la enajenación éstas siempre quedan en la esfera de lo ideal. (3)

La comprensión materialista no desestima el aspecto espiritual, pero busca su fundamentación última en la vida material de una sociedad concreta, en las relaciones sociales que le dan origen: la aparición de la división social del trabajo, que en el proceso del desarrollo social favorece la aparición de la propiedad privada sobre los medios de producción y desde entonces todas las formas de socialidad que se han sucedido expresiones del antagonismo social. Marx demostró al investigar en profundidad el capitalismo, cómo este tipo de sociedad intensifica hasta un punto extremo las contradicciones del proceso histórico, con el fin del incremento de las fuerzas productivas, creando la "forma extrema de alienación". (4)

No cabe dudas que con la existencia de la propiedad privada la sociedad mercantil toda se halla en condiciones favorables para la enajenación, que en los más diversos modos de manifestarse, se comporta como sociedad enajenada.

A través de la visión materialista sobre la enajenación se puede comprender su relación con la salud.

Durante siglos los hombres comprendieron la salud, como " norma" es decir lo opuesto a la enfermedad. De forma tal que si un individuo se correspondiera con la "norma" estaría saludable, pero no siempre que estuviera saludable se correspondería con la "norma". Entiéndase por "norma" los aspectos estrictamente biológicos.

Esta concepción fue superada por la definición de la Organización Mundial de la Salud (OMS) al señalar que: " La salud es un estado de completo bienestar físico, mental y social y no solamente la ausencia de las afecciones o enfermedades.

En su obra Tesis sobre Feuerback, "Karl Marx" definió la categoría salud refiriendo que la esencia humanista no es algo abstracto, inherente a cada individuo, es en realidad el conjunto de sus realidades sociales, y expresó la calidad de la existencia del hombre determinada por su relación armónica con el entorno natural-social que le corresponde.

De acuerdo con Barreras, A., Dujarric, R., y LLorens, J., la salud manifestándose como una interacción múltiple, debe definirse como: la calidad de la existencia del hombre determinada por su relación armónica con el medio social- natural que le corresponde.

La presencia de enajenación en la sociedad se relaciona directamente con la calidad de dicha existencia. En condiciones de alienación se limitan considerablemente los diversos 
componentes de la salud y se colocan junto a la ausencia de daños morfológicos y limitaciones funcionales otras cualidades de la salud como: determinado nivel de bienestar subjetivo, determinado nivel de bienestar social, lo que implica posibilidades sociales de desarrollo del individuo en el plano económico, cultural, etc.; y determinado nivel de desarrollo de la personalidad.

Resulta obvio que existiendo enajenación esos niveles que cualifican la salud se vean afectados provocando movimiento hacia los umbrales de enfermedad.

Según el modelo propuesto por Pérez Lovelle, existen tres niveles de determinación social de la salud, que son el nivel macro social, el nivel grupal y el nivel individual. (5)

\section{La enajenación y el nivel de determinación macro social de la salud.}

Este primer nivel es donde se establece relación entre la Formación Económico Social como un todo y el estado de salud de la población en general: ya sea del mundo, de una región o un país determinado.

La relación de la sociedad con la naturaleza en su etapa actual es una muestra evidente de cuán enajenada se halla la primera, en los marcos de un orden económico mundial que impone el capitalismo transnacionalizado.

La crisis ecológica que ha adquirido carácter global es el producto indeseado de una actividad humana desequilibrada y falta de perspectiva; es expresión de que en tales condiciones el hombre pierde en su esencia al poner en peligro su propia existencia y la vida toda en el planeta.

La influencia de la ecología en la salud es diversa y el hombre no puede pretender elevar la calidad de su existencia si no tiene en cuenta su relación con la naturaleza. Afortunadamente son muchas las voces que hoy reclaman cordura a nivel planetario en relación al problema ecológico, de la relación entre el desarrollo científico- técnico y el desarrollo social, y aparecen conceptos como el de desarrollo sustentable apuntando a la necesidad de no empeñar el futuro de las nuevas generaciones.

La abismal diferencia entre países llamados del primer mundo y los del mundo subdesarrollado muestra fenómenos que "mueven" hacia la enfermedad social y por ende son símbolos de enajenación, los males que genera la desigual distribución de las riquezas, tienen en la pobreza y sus secuelas para el estado de salud de las diversas poblaciones su primer exponente.

El desempleo y la marginalidad crecientes en el mundo contemporáneo pueden ser comprendidos tanto en el espacio interior de los países desarrollados, como de éstos con relación a los subdesarrollados. Si se tiene en cuenta la ley de depauperación de la clase 
obrera enunciada por Marx: mientras mayor riqueza, mayor pobreza se genera; eso significa mayor alienación de las clases marginales y desposeídas, entiéndase también mayor alienación en los países desposeídos.

Las diferencias en los cuadros de salud no dejan lugar a dudas, incluso entre regularidades al parecer más alejadas de la determinación económica, como la que existe entre las tasas de crecimiento de la población de una región a otra, y las diversas políticas de control de la natalidad que desencadenan.

La enajenación de este nivel macro social se expresa además en la propia actividad de Organización de la Salud Pública, analizada en tres vertientes:

- La alienación en la gestión de salud.

- La alienación en los profesionales de la salud.

- La alienación en las poblaciones con respecto a la salud.

La enajenación política- entendida bien como indiferencia política de las masas y como pérdida del sentido de dirección política a cuenta de interés mercantil en las condiciones del capitalismo, hoy constituye la fuente más directa de influencia sobre el extrañamiento de la salud pública.

El componente de propiedad privada en la salud pública existente en muchos países le imprime a dicha esfera un nítido carácter mercantil, aunque esté acompañado de formas estatales y condiciona la alienación de la gestión de salud, ésta en vez de enfrentar la situación de salud de acuerdo a necesidades sociales, persigue sólo fines económicos. Un fenómeno tal se ha producido con las trasnacionales farmacéuticas.

Es alienante la pretensión de cambiar el estado de salud de la población mediante cambios en los sistemas de salud, sin tener en cuenta que por m s que se cambien los mismos, la condicionante fundamental se halla en la esfera de lo económico, en las posibilidades de desarrollo económico del sistema.

Aún en el caso de tenerse en cuenta los indicadores que se utilizan por los Organismos Internacionales para evaluar el nivel de desarrollo humano y económico: esperanza de vida, distribución de ingresos, tasa de mortalidad infantil, etc., deben ser completados para la interpretación de las necesidades con otros tipos de variables esta vez subjetivas, de cómo el individuo percibe su vida y cómo se percibe así mismo. Sólo así se comprendería cómo un país desarrollado que exhibe un alto promedio de años de esperanza de vida por encima de los 75 , reporte la más alta tasa de suicidios. 
Por parte de los profesionales de la salud, la actividad se enajena cuando se aparta de su fin social y se convierte en medio de vida, eso se pone de manifiesto, sobre todo, donde dentro de la Salud Pública existen elementos de propiedad privada y el profesional partiendo de su situación de clase puede ser un asalariado o un propietario de cualquiera de las instituciones de salud. Este hecho no constituye algo absoluto, puesto que no dejan de existir los profesionales que aún en condiciones de propiedad privada, no apartan su actividad de su fin social. Mucho más cuando se trata de una esfera que exhibe una tradición ética profesional.

Del mismo modo, sería asegurar que no existe alienación en la profesión en una sociedad, por el hecho que no exista el elemento privado en la Salud Pública, no dejan de existir ejemplos de conductas inadecuadas. Un profesional que en un sistema de salud como el nuestro descuide la calidad del servicio, o que su fin sea el interés individual, el amiguismo o falta de responsabilidad, asumiendo actitudes alienadas.

Las manifestaciones de enajenación en la profesión contienen el factor subjetivo: concienciavaloración determinantes de la actitud a asumir, y que tal factor tiene a su vez una relativa independencia a las condiciones materiales de la actividad.

Analizando la población, las formas de enajenación con respecto a la salud son variadas, en primer lugar, las diferencias que existen entre los distintos países en cuanto al acceso a los servicios de salud y educación, ya en sí provocan grandes desbalances en los niveles de desarrollo de la cultura de la salud. En estos casos, la manipulación de las necesidades de salud de la población se da con abundancia marcada, cuando se persiguen fines económicos con los servicios, a través de los seguros médicos o con los medicamentos, por ejemplo.

\section{La enajenación y el nivel de determinación grupal.}

En este segundo nivel, la relación se establece entre el modo y las condiciones de vida de determinado grupo y el estado de salud de ese grupo ya sea clase, grupo profesional, etc.

Lo primero que salta a la vista es el hecho de que no hay una distribución homogénea de las enfermedades de todas las clases sociales de la población. Aunque no son comunes los estudios que lo demuestran, lo cierto es que las minorías, étnicas, las clases y los sectores desposeídos aún enferman de desnutrición, parasitismo e infecciones, técnicamente rebasadas por la medicina actual y por los niveles de vida que genera el desarrollo.

Pero lo más llamativo constituye que las propias condiciones de vida son las que determinan el lugar que ocupa la Salud en la jerarquía de valores de los grupos y clases y con ello sus actitudes con respecto a la enfermedad y la salud.

En el mundo capitalista contemporáneo la adhesión de los grupos marginales - en constante aumento - a la drogadicción, alcoholismo, prostitución y sus secuelas para la salud, forman 
parte del fenómeno enajenación que desvirtúa el aspecto humano de cualquier grupo social y que no le permite actuar consecuentemente con respecto a la salud.

Otro ejemplo que demuestra ese fenómeno sin que adquiera formas extremas es el caso de grupos ocupacionales a la manera de los que trabajan en la minería, que descuidan la protección del trabajo y con ello asumen posturas enajenadas con respecto a la salud. Claro que dichas posturas dependen en última instancia de la característica del sistema de producción en el que están incluidos.

\section{La enajenación y el nivel individual de determinación de la salud.}

Se trata de la relación entre el estilo de vida individual, las condiciones individuales de vida y el estado de salud también en el plano individual. La relación de este nivel con los dos anteriores proviene fundamentalmente, aunque no se limita a ello, del elemento condiciones individuales de vida, el que por supuesto está determinado directamente por las condiciones macro sociales y grupales, que en sí mismas portan el sustrato para un proceso individual de vida enajenado o no. Téngase en cuenta que en el individuo es donde se sintetizan las condiciones sociales de una formación económica determinada y las de la clase o grupo social a la que pertenece en particular.

Sin embargo, en la vida del hombre concreto junto a tales condiciones objetivas existen las que dependen del sujeto individual. Ambas se reflejan en el concepto Estilo de Vida: aspecto de la actividad diaria de las personas que indican la vida y orientación de la conducta y el racionamiento de la personalidad con una serie de variables que influyen en el proceso saludenfermedad dado por regulaciones volitivas que determinan las formas de satisfacer las necesidades básicas en dependencia de sus temores, conflictos, frustraciones, sentimientos, motivos, autovaloración, sexo, edad, escolaridad, actitudes, criterios, e imagen del mundo que tiene el individuo.

El grado de apropiación y asimilación del sujeto de sus condiciones existenciales depende del grado de desarrollo individual, de su personalidad, conocimientos, valores y actitudes, es decir, de su cultura. Cada individuo imprime un sello muy propio, un "estilo" con el que realiza esa apropiación de las condiciones económicas, políticas, morales y en el cual objetiva su existencia. Una peculiar forma de actuar.

Sin dudas que el estado de salud individual está en dependencia de las condiciones y del estilo de vida, donde entran en acción con un peso importante los elementos derivados del momento subjetivo de la actividad.

El hombre enajenado no puede asimilar correctamente sus condiciones de existencia y por tanto tampoco "elegir " adecuadamente su estilo de vida, sus fines. Se produce entonces un desbalance entre sus necesidades y capacidades y las posibilidades que tiene para 
satisfacerlas. "La salud es la existencia pletórica, de cuyo resultado la vida y la actividad del hombre son percibidas por él como autodesarrollo natural de las características y cualidades esenciales propias de él".

El desarrollo del hombre se mide pues por el nivel de desarrollo de sus capacidades y específicamente su capacidad de transformar el medio socio-natural, este enunciado coincide con la definición de calidad de vida que significa..." el grado de control que el individuo mantenga sobre su entorno."

El desarrollo de la personalidad, que es un elemento componente de la salud, es por tanto opuesto a enajenación.

Esencia y existencia alienadas, es decir, que el individuo está incapacitado de controlar el medio, que su vida no tiene la suficiente "calidad".

El estilo de vida escogido por el individuo tiene como ingrediente cómo se percibe por él la vida misma y con ello cómo se percibe y siente la salud, cómo se actúa con respecto a ella.

Conocimiento y actuación hacia la salud tienen como principio el lugar que estos ocupan dentro de la jerarquía de valores del individuo. Dicho lugar, si bien está determinado objetivamente por las condiciones materiales y espirituales: tradiciones, costumbres, también depende del sujeto mismo.

Es un ejemplo obvio de enajenación el que el individuo no se sienta y, por tanto, no actúe de forma responsable por su salud, y deposite la responsabilidad en terceros; ya sea concibiendo la salud como un "don" no terrenal que escapa a su dominio y la enfermedad un castigo, como depositando responsabilidad en el Estado (entiéndase los servicios de salud).

El fenómeno enajenación de la salud va mucho más allá: a la irracionalidad de la relación con la naturaleza, a la irracionalidad de las propias relaciones sociales:

entiéndase las relaciones de producción; a la organización de la salud pública y el carácter de los servicios de Salud; a la pertenencia de los individuos actuantes a determinados grupos y clases sociales y por último al propio individuo y su estilo de vida.

\section{Conclusiones.}

- El enfoque filosófico de la relación entre la enajenación y la salud tiene más de una arista de reflexión dado por la complejidad de ambos fenómenos, y contiene además una exigencia: la demostración de que tal enfoque existe y es necesario, sin caer en el peligro de incursionar en límites correspondientes a otras ciencias. 
- La Enajenación significa la limitación de la búsqueda por el hombre y adecuada elección del sentido de su vida

- La actitud de los hombres ante la salud es histórica - concreta, depende no solo de las condiciones sociales existentes, sino también de las valoraciones que poseen acerca de la misma, de sus conocimientos, costumbres, hábitos, condiciones de vida y del grado de satisfacción de sus necesidades vitales.

- En los modos de producción capitalistas la enajenación deja de ser un valor social para convertirse en un antivalor ya que el hombre se vuelve esclavo cada vez más y más de las cosas que produce y no satisface sus necesidades.

\section{Referencias bibliográficas.}

Macías Llanes María E. "LA RELACION ENTRE ENAJENACION Y SALUD", Proyecto Editorial CENDECSA, Universidad Médica Carlos J. Finlay, Camagüey, Cuba, 1999.

Quintero E y Alonso A. Ecología Agrícola. Edit. Pueblo y Educ. Cuba.1980.

Colectivo de autores cubanos. Lecciones de Filosofía Marxista-Leninista. Tomo 2: p. 372.

Marx, Carlos. "Fundamentos de la Crítica de la Economía Política 1857-1858", Vol. I, p. 481 (en ruso).

Macías Llanes María E. "LA RELACION ENTRE ENAJENACION Y SALUD", Proyecto Editorial CENDECSA, Universidad Médica Carlos J. Finlay, Camagüey, Cuba, 1999.

"Fronteras de la emancipación" (clases sociales a debate) en Actuel Marx, Universidad ARCIS, Santiago de Chile, 2004.

Colectivo de autores, Tomo II, Lecciones de Filosofía Marxista - Leninista

Enajenación y humanismo: aspectos de actualidad Lic.Georgina Suárez Hernández ( Consultado 6 de diciembre del 2019)

Honderich, Ted. Oxford Companion to Philosophy, Oxford: Oxford University Press, 2005.

La enajenación y su relación con el nivel individual de determinación de salud 2011 ilustrados.com, Monografias, tesis, bibliografias, educacion. Todos los temas y publicación ( Consultado 4 de diciembre del 2019)

Lecciones de Filosofía Marxista Leninista. Tomo 1 y 2. Editorial Félix Varela, La Habana, 1991.

Lecciones de Filosofía Marxista-Leninista. Colectivo de autores. MES, 1991:235-237. 
Lecturas de Filosofía, Salud y Sociedad Colectivo de Autores La Habana, 2000

Macías Llanes María E. "LA RELACION ENTRE ENAJENACION Y SALUD", Proyecto Editorial CENDECSA, Universidad Médica Carlos J. Finlay, Camagüey, Cuba, 1999. ( Consultado 4 de diciembre del 2019)

Marx, C. Manuscritos Económico-Filosóficos del 44. Ed. Pueblo y Educación, 1975:69-80.

Marx, Carlos. "Fundamentos de la Crítica de la Economía Política 1857-1858", Vol. I, p. 481 (en ruso).

Marx, Karl (1844). Manuscritos: Economía y Filosofía.

Marx, Karl (1845 -1846).Tomo I: Feuerbach. La Oposición entre idealistas y Materialistas. La ideología alemana.

Marx, Karl (1845-1846). La ideología alemana.

Selección de lecturas de los clásicos elaborada por los profesores de Filosofía y sociedad de la UNAH, 2015.

Teoría marxista de la alienación( Consultado 6 de diciembre del 2019)

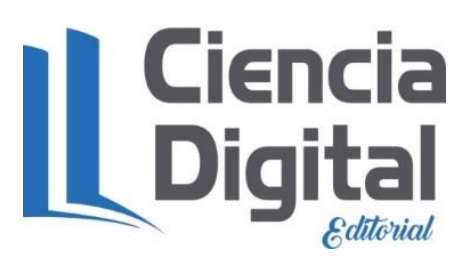




\section{PARA CITAR EL ARTÍCULO INDEXADO.}

Mederos González, A., Orta Espinosa, O., \& Martin Morell, D. E. (2019). La relación entre enajenación y salud teniendo en cuenta la propuesta de los tres niveles de determinación social de la salud. Anatomía Digital, 2(2), 6-17. https://doi.org/10.33262/anatomiadigital.v2i2.1076

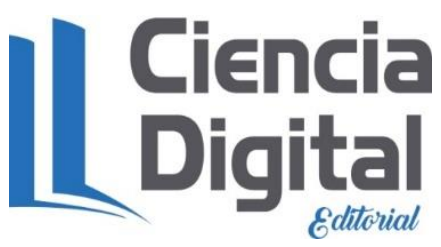

El artículo que se publica es de exclusiva responsabilidad de los autores y no necesariamente reflejan el pensamiento de la Revista Anatomía Digital.

El artículo queda en propiedad de la revista y, por tanto, su publicación parcial y/o total en otro medio tiene que ser autorizado por el director de la Revista Anatomía Digital.
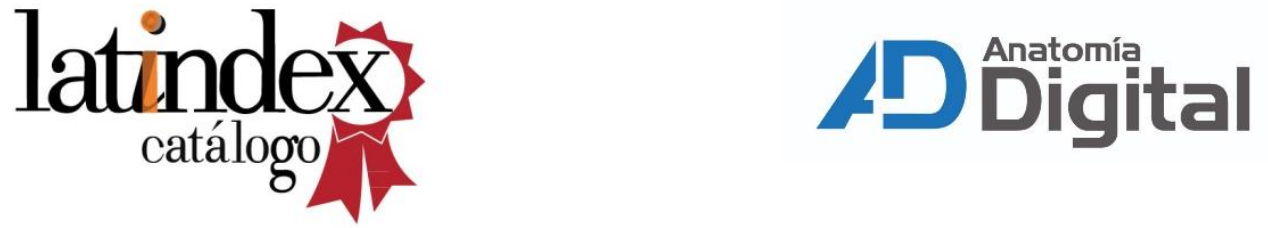